\title{
eJRIEPS
}

Ejournal de la recherche sur l'intervention en éducation physique et sport

15 | 2008

Varia

\section{Du savoir réellement enseigné en EPS au savoir de référence de l'enseignant : étude didactique clinique en karaté}

\section{Frédéric Heuser}

\section{(2) OpenEdition}

1 Journals

\section{Édition électronique}

URL : https://journals.openedition.org/ejrieps/5839

DOI : 10.4000/ejrieps.5839

ISSN : 2105-0821

\section{Éditeur}

ELLIADD

\section{Référence électronique}

Frédéric Heuser, « Du savoir réellement enseigné en EPS au savoir de référence de l'enseignant : étude didactique clinique en karaté », eJRIEPS [En ligne], 15 | 2008, mis en ligne le 01 juillet 2008, consulté le 03 mai 2021. URL : http://journals.openedition.org/ejrieps/5839 ; DOI : https://doi.org/10.4000/ ejrieps.5839

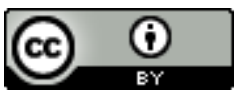

La revue eJRIEPS est mise à disposition selon les termes de la Creative Commons Attribution 4.0 International License. 
Du savoir réellement enseigné en EPS au savoir de référence de l'enseignant : étude didactique clinique en karaté

Frédéric Heuser

Professeur d'EPS au Département des APS de l'Université Toulouse I Sciences Sociales Doctorant au LEMME, EA 3692, Equipe AP3E, Université Paul Sabatier TOULOUSE III

\section{Résumé}

Cette thèse en cours se propose d'étudier en quoi une pratique enseignante renvoie à une référence et d'en déterminer la nature. L'objet de recherche se centre alors sur la recherche des traces du savoir de référence de l'enseignant, que nous allons extraire par l'analyse des écarts entre le savoir à enseigner, le savoir réellement enseigné, le savoir à évaluer et le savoir réellement évalué. La méthodologie s’appuie sur une étude didactique clinique, au cas par cas. L'analyse des verbatim d'un enseignant sur la première et la dernière séance d'un cycle de karaté en EPS, ainsi que des entretiens ante séance, post et d'après coup constituent le corpus qui va servir à extraire les traces du savoir enseigné et évalué dans le but d'inférer le savoir de référence de l'enseignant. Les premiers résultats sur une étude de cas montrent un constant remaniement du savoir de référence qui s'avère changer de nature au cours du processus d'enseignement.

Notre travail, en cours puisque la thèse n'a pas encore été soutenue, repose fondamentalement sur l'idée que l'enseignant se réfère toujours à quelque chose qui donne du sens à son enseignement. La référence est définie par le dictionnaire : « action de se rapporter à une chose, à un texte, à une autorité » (Quillet, 1985). En effet, « aucune recherche ne peut éviter, à un certain moment, la question de la référence, dans la mesure où le savoir transmis par l'enseignant se réfère le plus souvent à un déjà-là » (Terrisse, 2001). Nous postulons que le savoir de référence instaure un rapport à chaque étape de la transposition didactique et du traitement didactique opéré par l'enseignant, rapport auquel il ne peut échapper.

Le choix de l'activité karaté n'est pas innocent : tout d'abord il va sans dire que l'expertise du chercheur dans cet art martial a été le premier facteur de choix. Pratiquant depuis plus de vingt ans, professeur diplômé d'Etat et ceinture noire 3eme dan, nous avons notamment participé au développement du karaté en UNSS et avons enseigné de 
nombreux cycles à tous les niveaux de classe du collège et du lycée. Cette expertise permet donc de procéder à une analyse plus fine de ce que peuvent faire des collègues dans ce domaine. Le karaté est d'abord une activité bipartite : à la fois sport de combat avec une dimension sportive et moderne mais encore art martial avec sa dimension traditionnelle, d'art de la guerre, de méthode de combat et de formation de l'individu. Elle est en outre très éducative du fait des valeurs de maîtrise de soi, de respect et d'abnégation qu'elle véhicule. C'est ensuite une activité composée de styles différents (Shotokan, Shito ryu, Goju ryu, Kyokushinkai) qui sont parfois antinomiques. II s'avère en effet que ces écoles, bien qu'elles fassent toute partie de la famille karaté do, n'ont ni les mêmes fondements techniques, ni les mêmes approches stratégiques. En somme, les modes de transmission du savoir sont variables d'une école à l'autre dans la mesure où l'entraînement en club est plus orienté sur certains aspects que sur d'autres (qui le travail des katas dans une option traditionnelle, qui l'apprentissage des fondements du combat dans une option plus compétitive). Le karaté a enfin un statut «à part » : c'est avant tout une activité de spécialistes qui est peu enseignée et peu représentée dans le cadre des formations continues des enseignants d'EPS. II en découle une question capitale : à quel savoir l'enseignant se réfère-t-il lorsqu'il est confronté à l'enseignement d'une activité ne faisant ni l'objet de programmes, ni d'une didactisation avancée ? Nous sommes donc en présence d'un karaté pluriel. Cette pluralité va générer des expériences différentes chez les pratiquants et orienter les conceptions de l'enseignement à dispenser. De ce fait, les références vont être multiples et empreintes de l'expérience de pratiquant et d'enseignant et des conceptions de l'activité à enseigner.

Notre objet de recherche se centre alors sur la recherche de traces du savoir de référence (SR) de l'enseignant, que nous allons extraire par l'analyse des écarts entre le savoir à enseigner (SAE), le savoir réellement enseigné (SRE); le savoir à évaluer (SAEV); le savoir réellement évalué (SREV).

Cette quête des rapports en terme d'écarts entre SAE, SRE, SAEV et SREV nous amène à notre question de recherche : en quoi une pratique enseignante renvoie à une référence, et laquelle? Dans cette optique, nous posons comme hypothèse que l'analyse des rapports entre les différents savoirs va nous permettre d'inférer sur la nature (ce qu'il est) et le statut (ce qu'il représente) du savoir de référence de l'enseignant.

\section{Options théoriques}

Ce travail se situe dans le champ de la didactique clinique, qui articule des options théoriques spécifiques que nous allons préciser. Notre travail examine le processus de 
transmission des savoirs à partir du modèle de la ternarité qui est une formalisation du domaine des réflexions concernant la didactique. Trois éléments sont mis en relation: l'élève, l'enseignant et les savoirs. Ces trois éléments constituent les trois pôles d'un triangle que l'on appelle le triangle didactique. La mise en relation de ces trois pôles est appelée « système didactique » par Colomb, qui est «formé par trois éléments : l'enseignant, les élèves, le savoir et la relation ternaire qui les lie » (1986). L'objet d'étude de la didactique clinique s'intéresse plus précisément à ces mises en relations dans le but d'une meilleure compréhension du fonctionnement didactique. Notre problématique nous amène à nous concentrer sur les influences qui s'établissent entre deux pôles du triangle : l'enseignant et le savoir qu'il transmet (transposition didactique). En effet, importé dans le champ spécifique de la didactique clinique, ce temps du processus de transposition est revisité par la prise en compte de la dimension du sujet ou pour reprendre des auteurs comme Beillerot, Blanchard-Laville et Mosconi en 1996 «le sujet singulier dans sa dynamique psychique et sociale ». L'approche clinique en didactique s'appuie sur une théorie du sujet didactique qui comme l'a formalisé M.F. Carnus (2004) peut être « singulier, assujetti ou divisé ».

Nous utilisons en premier lieu le concept de la transposition didactique, initié par Verret (1975) et développé par Chevallard, et nous le mettons au cœur de notre problématique dans la mesure où nous le considérerons de manière synthétique comme « l'ensemble des transformations que fait subir à un champ culturel la volonté de l'enseigner dans un cadre scolaire ». (Chevallard, 1985). Ce processus va permettre la construction de la pratique sociale de référence karaté afin de pouvoir l'enseigner à l'école, en EPS, selon trois étapes (Terrisse, 1998) :

« Du savoir de référence au savoir à enseigner » (choix de l'enseignant en fonction notamment de sa conception de l'activité).

« Du savoir à enseigner au savoir enseigné » (mise en œuvre du savoir à travers les contenus d'enseignement).

« Du savoir enseigné au savoir appris » (contrat didactique entre enseignant et élèves).

Nous nous attacherons surtout aux deux premier temps, à savoir les passages du savoir de référence au savoir à enseigner, puis du savoir à enseigner au savoir enseigné. Dans le cadre de notre problématique de recherche, nous modifierons la troisième et dernière étape, car notre étude part du savoir enseigné, non pour remonter au savoir appris comme nous l'avons déjà réalisé dans une autre étude (Heuser, 2005), mais pour remonter au savoir qui a servi de référence à l'enseignant, retour en boucle qui pose la question des influences qui sous-tendent aussi bien les intentions que les décisions de l'enseignant. 
Cela va exiger du chercheur un véritable «profilage » des différents temps de l'enseignement (SAE, SRE, SAEV, SREV). Si les influences à tous ces niveaux vont être nombreuses, c'est aussi parce que les références le sont : C. Raisky (1993) envisage bien que « dans un système didactique, les références sont multiples ». Elles peuvent être en effet externes (tout ce qui est extérieur à l'école), ou internes (tout ce qui est du fait de l'école). Nous rajouterons qu'elles peuvent être aussi personnelles, dans la mesure où les enseignants se construisent des références qui leur sont propres. C'est aussi ce qui fait dire à Caillot que « la question de la référence se pose en fait pour chaque pôle (du triangle didactique: sujet, élève, savoir). Au lieu de parler d'une seule référence, nous devons penser à des références multiples qui doivent concerner l'ensemble du triangle » (Caillot, 2001).

La question de la référence est donc centrale. Martinand (1981), s'il partage le questionnement qu'introduit la transposition didactique, celle de rendre compte de l'origine et de la transformation du savoir pour qu'il soit enseigné, pour lui, cette opération correspond à une «composition sous influences» (2000), sociales, politiques, idéologiques, pédagogiques.

II ne faut pas oublier à ce propos que l'EPS est une discipline "à support pratique" et non pas "à support théorique" comme les mathématiques. Elle dispense des "pratiques corporelles" par le biais d'Activités Physiques et Sportives (APS). Comme en EPS la référence est multiple, les savoirs à enseigner se construisent à partir de diverses références (cf. figure 1):

- Référence à la pratique sportive et aux savoirs techniques attenants (expérience de pratiquant).

- Référence aux savoirs scientifiques gravitant autour de cette pratique (conception de l'activité).

- Référence à l'enseignement et aux savoirs professionnels mis en jeu (expérience d'enseignant).

Les références sont donc multiples dans la mesure où tout enseignant a des savoirs d'experts (référence culturelle), des savoirs d'expérience (référence personnelle et sociale) qu'il doit prendre en compte dans un contexte institutionnel, et il est vrai que dans le cas du karaté, c'est le vide institutionnel, puisqu'il n'y a aucune référence au karaté dans les programmes d'EPS et que l'enseignant est contraint de s'inspirer soit de la boxe française, soit de la lutte. 
Pratique sportive et savoirs techniques

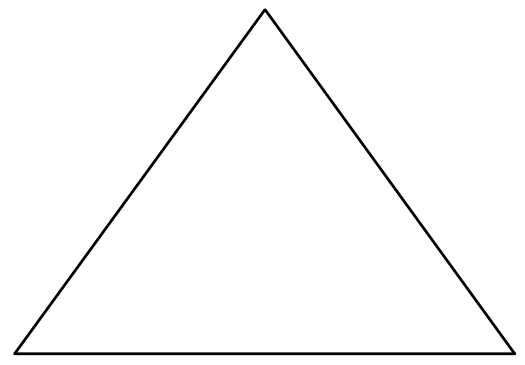

Savoirs

Savoirs

scientifiques

professionnels

Figure 1. Les savoirs de référence (Terrisse, 2001)

Ces options théoriques rejoignent les auteurs qui comme Charlot (1997) se demandent « en quoi la référence intègre l'étude du sujet dans son rapport au savoir ». Dans ses travaux, le rapport au savoir est étudié au niveau psychosociologique: «Analyser le rapport au savoir, c'est étudier le sujet confronté à l'obligation d'apprendre dans un monde qu'il partage avec d'autres: le rapport au savoir est rapport au monde, rapport à soi, rapport aux autres » (ibid.). Cette dimension sociale du rapport au savoir renvoie aussi au rapport à l'institution. C'est ce que développe Chevallard, dans son approche globale du phénomène, plus didactique et anthropologique : « un individu concret ne peut entrer en rapport avec un savoir qu'en entrant en relation avec une ou des institutions " (Chevallard, 1989). En effet, l'auteur distingue notamment trois types de rapports aux savoirs : un rapport personnel d'abord, que l'on qualifiera d'intime à l'enseignant, un rapport officiel qui renvoie lui à son statut et enfin un rapport institutionnel car il exerce dans un contexte donné avec un cadre particulier qui est par exemple l'assujettissement aux programmes. Le rapport au savoir s'exerce donc à plusieurs niveaux, comme le détaillent $A$. Terrisse et de J.P. Sauvegrain (1998) : « le savoir à enseigner est le rapport entre l'enseignant et son savoir sur l'activité » et « le savoir enseigné est le rapport entre le savoir précédent et la mise à l'épreuve de l'enseignement face aux élèves ».

\section{Options méthodologiques}

Du point de vue méthodologique, l'étude se fait au cas par cas. Comme le souligne A. Terrisse (2001) : «la clinique sert à interroger le rapport singulier qu'entretient le sujet à l'activité qu'il pratique pour en rendre compte, l'identifier, la caractériser ». Ce rapport « ne pourra être élaboré qu'à partir d'une prise en compte du cas par cas, ce qui caractérise la 
clinique du singulier, celle de la position subjective de chacun ». Dans sa méthode, elle s'attache aux pratiques ordinaires, non prescriptives et intègre l'apport de preuves, qui renvoient aux traces que l'on cherche dans toutes les communications de l'enseignant, singulières et personnelles (écrites dans les préparations de leçons et orales au cours même de celles-ci ainsi que des entretiens), au cours des différentes phases de la méthodologie qui sera développée plus après : le déjà-là, l'épreuve (l'acte d'enseignement proprement dit) et l'après coup.

\section{1. Méthodologie de recueil des données}

Nous avons procédé à une analyse clinique du discours du professeur. Autrement dit, les communications de l'enseignant sont analysées au cours des trois temps de la clinique : le déjà là (planifications, entretien ante séance), l'épreuve (l'acte d'enseignement et l'après coup (entretiens post séance et d'après coup). Afin de rendre compte de la dimension temporelle si essentielle dans ce processus d'analyse, Terrisse, Carnus et Sauvegrain ont synthétisé les trois temps du recueil des données en didactique clinique sous la forme du tableau suivant (tableau 1) :

Tableau I. Les trois temps du recueil des données (Terrisse, Carnus, Sauvegrain, 2002)

\begin{tabular}{|l|l|l|l|}
\hline Temps & Déjà-là & Epreuve & Après coup \\
\hline Enseignant & Planification & Mise en oeuvre & Remaniement \\
\hline
\end{tabular}

L'enregistrement audio et vidéo de l'enseignant d'EPS porte sur la première et la dernière séance du cycle. Nous avons ainsi accès à l'entrée dans l'activité, aux savoirs en jeu dans le cycle et aux savoirs évalués. Comme l'étude de cas porte essentiellement sur le verbatim, notre corpus est constitué à partir de l'outil audio, retranscrit soit à partir du dictaphone porté par l'enseignant, soit à partir du son enregistré avec l'image vidéo. En effet, la vidéo nous sert surtout à contextualiser le discours de l'enseignant, pour savoir par exemple s'il s'adresse à un moment de la leçon à un élève ou à un groupe d'élèves. A chaque étape, nous réalisons un entretien ante séance, un post séance et enfin un entretien d'après coup. La technique de recueil des données est l'entretien semi dirigé. Celui-ci sera réalisé en début de chaque séance (entretien ante séance), puis à la fin de celles-ci (entretien post séance). Ces entretiens semi dirigés ante séance et post séance nous ont permis de recueillir les intentions didactiques des professeurs d'une part, leurs interprétations de l'enseignement dispensé au cours de chaque séance d'autre part. Nous avons aussi questionné dans l'après-coup, car comme le souligne A. Terrisse (ibid.) : «à la manière des cliniciens, nous postulons que seul le sujet peut rendre compte de ses 
actes ». Les entretiens sont tous semi dirigés afin de laisser une part importante d'expression libre et non contrainte à l'enseignant.

Ainsi, au niveau méthodologique, les entretiens ante ont pour but de fournir des informations sur le déjà là, sur le choix des savoirs à enseigner et à évaluer et les effets attendus par les enseignants en termes d'acquisition de savoirs par les élèves. C'est pourquoi nous interrogeons en premier lieu l'enseignant sur son expérience du karaté (pratique et enseignement), dans l'entretien ante séance 1. Puis nous posons une question sur ces intentions lors de la séance et plus généralement du cycle. Enfin, nous revenons sur un aspect du projet de cycle fourni par l'enseignant si quelque chose ne paraît pas clair.

Au niveau des entretiens post, la première question demande à l'enseignant de faire un bilan de sa séance. Puis nous l'interrogeons sur l'écart entre le projet de séance fourni et ses réalisations effectives. Enfin, les questions suivantes vont concerner l'entrée dans l'activité, et des précisions sur certaines situations proposées. Dans les entretiens d'après coup, nous revenons avec l'enseignant sur les écarts repérés. Le plus souvent, nous le faisons en mettant en contradiction un extrait de verbatim avec une réalisation effective au cours d'une des séances.

\section{2. Méthodologie de traitement des données}

Le traitement des données comporte quatre temps, au cas par cas. Le premier temps consiste en une lecture sélective des réponses de l'enseignant à l'entretien ante séance et aux planifications (cycle, séance). Cette phase nous donnera des éléments sur le SAE et le SAEV. Au cours du deuxième temps, nous analysons le SRE et le SREV, au travers des verbatim des deux séances enregistrées et des entretiens post séance. Le troisième temps est consacré au pointage des écarts entre SAE, SRE, SAEV et SREV. C'est au cours de cette phase que seront sélectionnés des extraits de verbatim significatifs de ces écarts afin de préparer l'entretien d'après-coup. Des hypothèses sur le SR de l'enseignant pourront être alors suggérées par le chercheur lors de cette phase. Le quatrième et dernier temps permettra de confirmer les hypothèses concernant le SR de l'enseignant, en confrontant celles-ci aux réponses de l'enseignant dans l'entretien d'après coup alors réalisé. Nous pourrons lors de cette phase établir un réel " profil » du savoir de référence de l'enseignant.

Le tableau suivant (tableau II) se propose de récapituler ces quatre phases de notre méthodologie de traitement des données : 
Tableau II. Les quatre phases du traitement des données

\begin{tabular}{|c|c|c|c|}
\hline \multicolumn{4}{|c|}{ LES QUATRE PHASES DU TRAITEMENT DES DONNEES } \\
\hline PHASE 1 & PHASE 2 & PHASE 3 & PHASE 4 \\
\hline $\begin{array}{l}\text { Repérage SAE et } \\
\text { SAEV }\end{array}$ & $\begin{array}{l}\text { Repérage } \\
\text { SRE et SREV }\end{array}$ & Pointage des écarts & Détection du SR \\
\hline $\begin{array}{l}\text { Entretiens ante } \\
\text { Planifications cycle } \\
\text { et séances }\end{array}$ & $\begin{array}{l}\text { Analyse des } \\
\text { séances : extraction } \\
\text { et mise en évidence } \\
\text { des différents } \\
\text { savoirs enseignés et } \\
\text { évalués }\end{array}$ & $\begin{array}{l}\text { Hypothèses sur le } \\
\text { SR }\end{array}$ & $\begin{array}{l}\text { Conclusions de } \\
\text { l'étude clinique }\end{array}$ \\
\hline & Entretiens post & & \\
\hline
\end{tabular}

\section{Résultats d'une étude de cas : David}

3. 1. Le SAE : ce que David dit vouloir faire

David dit au cours de l'entretien ante séance 1 que les séances sont " basées essentiellement sur un apport technique au départ avec glissement progressif vers l'assaut et le combat ». II s'avère que David accorde une place non négligeable aux apports purement techniques, mais en début d'apprentissage, après quoi il va pouvoir passer à un enseignement plus stratégique et tactique, une fois que les bases techniques sont intégrées par les élèves. Toujours dans le même entretien David dira : "Ensuite, il y a l'aspect je dirais technico-tactique, travailler sur l'enchaînement pieds-poings, c'est la première transformation que je souhaite voir chez les élèves: passer d'un travail de poings unique à un enchaînement pieds-poings ». En conclusion, on peut dire que le SAE de David se positionne clairement dans une optique d'articulation duo/duel, qui est d'ailleurs prônée par les programmes dans les activités de combat en lycée. Le SAE de David se révèle dans une dominante que l'on qualifiera de technico-tactique.

3. 2. Le SRE : ce que David fait réellement et dit réellement faire

Dès le début de la première séance David met en place un travail qu'il qualifie lui de technique : «Bien, il se met en garde. Moi je me mets ici et je vais faire partir...Vous partez d'ici, vous armez. Concentration première chose sur les appuis. Les sensations des appuis sont importantes, abdos fessiers contactés. C'est de l'énergie, on la restitue. Là on verrouille et c'est lourd ». Pour autant, cette situation met en jeu pour nous plus que des 
savoirs techniques. David n'est pas dans la technique pure dans la mesure où il ne s'agit pas de répéter un mouvement de manière décontextualisée. Dans cette situation, les élèves sont en effet par deux, dans un contexte de travail en duo. II y a une cible à viser, et des consignes sur les appuis, les sensations sont données par l'enseignant. Même si les élèves ne sont pas en déplacement, ils doivent ajuster leur distance par rapport au partenaire afin de délivrer le coup le plus efficace possible. David donnera dans l'entretien post séance sa définition de la technique, et il est vrai qu'elle est en accord avec le travail qu'il fait réaliser aux élèves : " Je suis rentré par la technique pour travailler justement sur la sensation, sur l'assise, pour dédramatiser un peu le combat ». David utilise alors des savoirs techniques dans le but d'atteindre des objectifs d'ordre affectif.

Nous reviendrons avec David sur ce point dans l'entretien d'après coup et il sera très clair sur le terme à utiliser : pour lui, il s'agit là d'un apprentissage " proprioceptif ». Ce travail renvoie plus à une «démarche d'apprentissage » qu'à un "objectif sécuritaire ou informationnel ». Pour autant, l'enseignement de David lors de cette première séance est aussi empreint de savoirs sécuritaires. Dans la présentation aux élèves du cycle David leur dit: " Ce qui est intéressant pour nous, ce n'est pas de se mettre K.O. Comprenez bien que ce soit mon rôle aussi bien que votre intérêt à vous, c'est que tout le monde pratique en toute sécurité ». David intègre donc progressivement à son enseignement des savoirs sécuritaires de manière aussi à développer l'autonomie de ses élèves en les responsabilisant sur les règles à respecter comme par exemple les zones cibles autorisées en combat : "Nous pratiquerons des touches essentiellement, pas des frappes. Nos zones cibles seront épaules, pas de coups au visage. C'est un sport qui se travaille à mains nues, mais quand on fera des combats, nous utiliserons quand même des gants de boxe, pour des raisons de sécurité ». Pour lui, la maîtrise technique est un facteur essentiel de sécurité et il l'évoquera clairement dans l'entretien post séance : " au karaté si la technique n'est pas un minimum assise ou acquise par les élèves, on touche à des problèmes de sécurité. Dans le combat, avec la gestion affective de l'activité, des techniques qui ne sont pas suffisamment maîtrisées peuvent amener des blessures inutiles, que l'on peut éviter en travaillant comme on le fait sur des sensations, sur des placements, sur des cibles. Cela permet de limiter les risques d'accident». David reviendra d'ailleurs sur cet aspect lors de l'entretien post séance d'évaluation, ce qui prouve bien l'importance de cette préoccupation pour lui : «Je me refuse à faire du combat sur des aspects techniques qui ne sont pas maîtrisés parce qu'en combat il faut que les élèves puissent travailler en confiance et s'ils commencent à se faire mal parce que les techniques ne sont pas suffisamment maîtrisées, on arrive à annihiler tout le 
bénéfice et l'intérêt du combat. II semble que David a vraiment ce souci de sécurité, que l'on peut peut-être expliquer par le fait de son choix de l'école de référence kyokushinkaï, réputée pour son efficacité, et même sa dureté. L'enseignant a donc voulu se prémunir au maximum de possibles problèmes liés au respect de l'intégrité physique de ses élèves, comme il peut en vivre en club ce qu'il ne souhaite pas voir émerger dans son cours d'EPS. Le SRE de David durant cette première séance est donc à dominante technique et sécuritaire. En effet, David ne conçoit pas la technique comme une fin (d'apprentissage d'un seul geste) mais comme un moyen (de contrôle du geste dans une optique sécuritaire). On est en effet sur la recherche de constructions de perceptions, de sensations mais on s'aperçoit que c'est le statut même du savoir qui change. On peut dans le cas de David inférer une référence de nature sécuritaire, informationnelle et affective.

\section{3. Le SAEV : ce que David dit vouloir évaluer}

Les intentions de David en matière d'évaluation sont énoncées par lui de manière brève. II a fourni la fiche d'évaluation dont les élèves vont se servir, sans la détailler. II dira juste que les élèves sont évalués en combat, en co-arbitrage et co-évaluation avec en défense des critères de " mobilité », " de garde et de décalage »; en attaque: "les cibles, l'enchaînement et précision des coups ".

3. 4. Le SREV : ce que David évalue réellement et dit réellement évaluer

David évalue ses élèves dans l'épreuve du combat libre. Dans l'entretien post, David évoque le contenu de son évaluation : "même si les critères d'évaluation sont techniques, ce qui est important, en combat, c'est d'y voir clair. C'est-à-dire que ça sert à rien d'aller dans le combat avec des coups qui ne sont pas maîtrisés, qui ne sont pas portés à distance ». C'est pour cette raison que la fiche d'évaluation comporte des aspects techniques : "garde, se protège; enchaîne ses coups ». L'aspect stratégico tactique de l'épreuve est avéré, puisque chaque élève est évalué en attaque et en défense et de surcroît va rencontrer des adversaires différents et de niveau sensiblement équivalent ( Au niveau du combat, on va travailler au moins sur trois assauts avec des partenaires de compétence et d'implication similaires dans l'activité ». Cf. Entretien post séance 1). David, quand il revient sur la prestation de ses élèves à l'évaluation est d'ailleurs positif dans ses retours : "Je ne pensais pas qu'on arriverait rapidement à avoir des combats relativement propres, avec des stratégies de tourner, d'occuper l'espace ». Dans l'entretien post, on peut lire aussi : «j'ai trouvé ça assez intéressant (...) on est arrivé à des sorties dans le dos, des décalages, des impacts. Pour moi les écarts les plus sensibles se situeraient au niveau des appuis, au niveau de la précision où là on n'est pas 
tout à fait rentré dans les objectifs. Par contre, les stratégies de défense, de déplacements, d'adaptation des coups ou de l'arme à la distance, là je pense qu'il n'y a pas d'écart. C'est très positif ". On peut renvoyer là à ce que disait David sur l'importance en combat d'y voir clair. En effet, pour David, «y voir clair » c'est s'adapter au niveau stratégique, en terme d'arme, de déplacement et de distance. On est bien loin de critères purement techniques.

De ce fait, le SREV de David est essentiellement stratégique, la technique est évaluée mais comme preuve d'atteinte de compétences plus stratégiques.

3. 5. Analyse des écarts SAE-SRE-SAEV-SREV et hypothèses sur le SR.

Tableau III. Récapitulatif des écarts aux savoirs enseignés et évalués de David $\underline{X}$ : dominant $X$ : secondaire

\begin{tabular}{|l|l|l|l|l|}
\hline SAVOIRS & SAE & SRE & SAEV & REV \\
\hline Techniques & $\underline{\mathrm{X}}$ & $\underline{\mathrm{X}}$ & $\mathrm{X}$ & $\mathrm{X}$ \\
\hline Stratégiques & $\mathrm{X}$ & $\mathrm{X}$ & $\underline{\mathrm{X}}$ & $\underline{\mathrm{X}}$ \\
\hline Sécuritaires & & $\underline{\mathrm{X}}$ & & $\mathrm{X}$ \\
\hline
\end{tabular}

L'analyse clinique du cas David montre une forte dominante technico tactique de son enseignement et de son évaluation (cf. tableau 3). Les aspects techniques de son enseignement lui permettent en effet de rentrer dans l'activité en toute sécurité et lui donnent un fil conducteur qu'il n'abandonne jamais totalement, même à l'évaluation. Par contre, l'aspect stratégico tactique est occulté lors de la première séance, ce qui s'explique par l'intention de David de "sécuriser" les savoirs en jeu en début d'apprentissage et de mettre les élèves en confiance par rapport à ceux-ci pour la suite du cycle. David choisit d'évaluer les élèves sur des aspects uniquement stratégiques, dans l'épreuve du combat, car pour lui la technique est plus un moyen qu'une fin. De plus, comme il a été particulièrement satisfait de la progression des élèves au cours du cycle (cf. entretien d'après coup), les évaluer en combat est un objectif qu'il a pu atteindre. Malgré tout et même s'il s'explique, on observe d'un point de vue théorique une rupture entre le savoir enseigné et le savoir évalué. Comment rentrer comme le fait David par la technique et en sortir par le combat? Pourquoi alors ne pas rentrer directement par le combat ? Ces questions ont été posées à David dans l'entretien d'après coup : il répondra qu'il ne rentre pas par le combat pour des questions de sécurité uniquement. Ce n'est pas «sa démarche d'apprentissage » (cf. entretien d'après coup).

Nous avions dans les analyses préalables, associé le profil de David à « la tradition ». Après l'étude clinique qui a été faite, nous dirons que son profil s'associe plus à « l'efficacité ». Ceci est sans doute le fait de la nouvelle forme de karaté qu'il pratique au 
moment de l'expérimentation, le kyokushinkaï. Cette école est en effet centrée sur l'efficacité en combat, préoccupation que l'on retrouve chez David dans l'entretien post séance 1 : "L'intérêt de cette activité kyok c'est que là on touche à l'efficacité, ce qui va faire que l'activité va être crédible ».

\section{6. Conclusions de l'étude de cas : le savoir de référence de David}

La référence de David s'avère au regard des analyses précédentes d'abord technique, pour garantir le respect de l'intégrité physique de ses élèves. Dans le même ordre d'idée et pour reprendre un terme utilisé par David lui-même, cette référence est " culturelle » : " c'est un passage qui est plutôt culturel, qui est le kihon et je garde ici dans mon enseignement cet aspect traditionnel ॥ (cf. verbatim post séance 1). Pour autant, David se réfère aussi à des aspects stratégiques puisqu'il a la volonté d'articuler duo et duel progressivement tout au long du cycle, pour aboutir d'ailleurs à une évaluation centrée sur des aspects stratégiques : le combat. Or, le combat est considéré comme l'essence même du karaté ou de tout autre art martial. Le karaté est en effet, un art de guerre, de préparation au combat, dans une optique d'auto défense. C'est donc aussi un aspect culturel du karaté que David intègre à son enseignement.

\section{Conclusion}

A ce stade de notre recherche, les premiers résultats font état de la dynamique de la nature de la référence utilisée par l'enseignant : elle se révèle bien dans les écarts mais parce qu'elle est constamment remaniée par l'enseignant au cours des différentes phases de son enseignement. En définitive, il s'avère que c'est moins la référence que le statut de cette référence qui change dans ces remaniements. Dans le cas de David le savoir de référence est en effet technico tactique mais sa nature change, passant d'une nature sécuritaire à informationnelle, proprioceptive.

II semble alors que l'écart lui-même devient un outil d'analyse de tout premier ordre au chercheur car il lui révèle le statut de la référence: utile ou cachée. Cette conclusion questionne son rapport personnel aux savoirs qu'il dit avoir comme référence ainsi que la part de l'insu dans les remaniements de la référence. En définitive, la référence s'avère avoir une part non négligeable dans l'organisation de la pratique enseignante. De ce fait, elle permet non seulement de prendre en compte les savoirs en jeu mais surtout d'en rendre compte et d'aborder le problème de certaines difficultés d'enseigner en posant la question des rapports aux savoirs de l'enseignant, domaine en cours d'exploration dans le champ des sciences de l'éducation, et que les travaux de notre équipe de recherche tentent d'élucider. 


\section{Bibliographie}

Carnus, M. F. (2003). Croyances, conceptions, intentions et pratique usuelle dans l'enseignement de la gymnastique : le cas de l'ATR et du repérage, dossier EPS $n^{\circ} 57$, Paris, Editions Revue EPS.

Charlot, B. (1997). Du rapport au savoir. Eléments pour une théorie. Paris: Anthropos (Editions Economica).

Chevallard, Y. (1985). La transposition didactique. Du savoir savant au savoir enseigné. Grenoble : La Pensée sauvage.

Heuser, F. (2005). Ecart entre savoir enseigné et savoir appris en EPS : étude de cas en karaté. In Revue e-jrieps (e-journal de la recherche sur l'intervention en EPS, édité par l'IUFM de Franche Comté).

Loizon, D. (2004). Analyse des pratiques d'enseignement du judo : identification du savoir transmis à travers les variables didactiques utilisées par les enseignants en club et en EPS. Thèse de doctorat en sciences de l'éducation, Université Paul Sabatier Toulouse III.

Martinand, J.L. (1981). Pratiques sociales de référence et compétences techniques. A propos d'un projet d'initiation aux techniques de fabrication mécanique en classe de quatrième, in A.Giordan (coord.). Diffusion et appropriation du savoir scientifique: enseignement et vulgarisation. Actes des Troisièmes Journées Internationales sur l'Education Scientifique. Paris-Bruxelles : De Boek Université.

Martinand, J.L. (2000). Pratique de référence et problématique de la référence curriculaire, in A. Terrisse (Eds) Didactique des Disciplines: les références au savoir. Bruxelles : De Boeck, Perspectives en éducation.

Quillet/Flammarion (1985) : dictionnaire usuel. Paris, Editeurs Quillet-Flammarion

Raisky, C. (1993). Problème du sens des savoirs professionnels, préalable à une didactique in sens des didactiques et didactique du sens. Editions du CRP: Sherbrooke.

Sauvegrain, J.P. (2001). Analyse didactique de la décision de l'élève, étude de cas sur l'utilisation du savoir dans un cycle de lutte en Education Physique et sportive. Thèse non publiée, université Paul Sabatier Toulouse III.

Terrisse, A. (2001). Didactique des disciplines: les références au savoir. Bruxelles: De Boeck Université. 
Terrisse, A., \& Sauvegrain, J.P. (1998). Identification des difficultés d'appropriation du savoir lutter par une méthodologie d'ingénierie didactique. In Revue Science et motricité $\mathrm{n}^{\circ}$ 32-33.

Terrisse, A. (1995). Savoir combattre : essai d'élucidation, Revue EPS 252, mars-avril.

Van Der Maren, J.M. (1996). Méthodes de recherches pour l'éducation. De Boeck éditions.

Verret, M. (1975). Le temps des études. Thèse présentée devant l'Université de Paris V. Librairie Honoré Champion (diffusion). 